\title{
Pédagogie universitaire et classe inversée : vers un apprentissage fructueux en travaux pratiques
}

Fatima Lakrami, Ouidad Labouidya et Najib Elkamoun

\section{OpenEdition}

Édition électronique

URL : https://journals.openedition.org/ripes/1793

DOI : 10.4000/ripes.1793

ISSN : 2076-8427

Éditeur

Association internationale de pédagogie universitaire

Référence électronique

Fatima Lakrami, Ouidad Labouidya et Najib Elkamoun, «Pédagogie universitaire et classe inversée vers un apprentissage fructueux en travaux pratiques », Revue internationale de pédagogie de l'enseignement supérieur [En ligne], 34(3) | 2018, mis en ligne le 20 novembre 2018, consulté le 21 septembre 2021. URL : http://journals.openedition.org/ripes/1793 ; DOI : https://doi.org/10.4000/ ripes. 1793

Ce document a été généré automatiquement le 21 septembre 2021.

Article L.111-1 du Code de la propriété intellectuelle. 


\title{
Pédagogie universitaire et classe inversée : vers un apprentissage fructueux en travaux pratiques
}

\author{
Fatima Lakrami, Ouidad Labouidya et Najib Elkamoun
}

\section{Introduction}

1 L'enseignement supérieur demeure depuis toujours au cœur de tous les débats relatifs à l'éducation partout dans le monde, il est le centre de plusieurs problèmes et débats (Prost, 1986): massification, démocratie, ... Il a de plus en plus un rôle de levier essentiel de développement surtout dans une économie du savoir basée sur la recherche et l'innovation. Il s'est transformé au cours des deux dernières décennies sous l'effet de la globalisation économique et financière et aussi l'arrivée des nouveaux avancements technologiques, on commence déjà à se demander sur sa continuité probable sans les TIC (Vétois, 2016). En effet, au cours des vingt-cinq dernières années, de nombreux pays ont développé des mécanismes de garantie de la qualité de l'enseignement universitaire à la base des nouvelles technologies innovatrices (Vindevoghel et Blondeau, 1998).

Dans cette lignée, les établissements universitaires marocains sont amenés à relever plusieurs défis et à repenser les modalités de l'enseignement/apprentissage pour favoriser une plus grande participation des étudiants et promouvoir des pratiques pédagogiques innovantes. Vu la croissance des effectifs et la limitation des moyens d'encadrement, une telle évolution conduit à relever simultanément des challenges pédagogiques, technologiques et organisationnels. L'université Marocaine doit donc réussir à faire face au bouleversement structurel des nouveaux modes d'accès, de création et de diffusion des savoirs. Elle doit penser à suivre le train d'une telle évolution qui s'inscrit sous le volet d'une transition partielle vers le « tout numérique ». De telles actions ont comme principal objectif le développement de nouvelles formes 
d'apprentissage actif et collaboratif s'appuyant sur des outils de cours nomades et omniprésents.

\section{Problématique et objectif}

3 En général, les Travaux Pratiques (TP) sont considérés comme une partie essentielle de l'enseignement et de l'apprentissage de la science (Pekmez, Johnson et Gott, 2005 ; Abrahams et Millar, 2008; Abrahams, Reiss et Sharpe, 2013). Cette position a été confirmée par des chercheurs, enseignants ainsi que des programmes d'études internationaux (Dillon, 2008). Par ailleurs, on considère que généralement les étudiants semblent apprécier le travail pratique et cela accroit leur motivation à étudier la science. En effet, de nombreux chercheurs ont constaté que l'apprentissage de la science ainsi que le niveau de compréhension sont améliorés lorsque les étudiants sont engagés dans des expériences pratiques au sein d'un laboratoire scientifique (Hofstein, 2004 ; Lunetta, Hofstein et Clough, 2007). Dans ce sens, le sociologue Edgar Morin (1984) a même affirmé que «La Science est un mode de connaissance fondé sur le dialogue entre les théories et les données observées ou issues de l'expérimentation ». Les résultats des sciences ne sont donc pas à enseigner comme des données à croire mais comme des résultats produits par des méthodes expérimentales. «Les savoir-faire expérimentaux ne peuvent être acquis par les élèves que si ceux-ci ont manipulé durant des séances de travaux pratiques. »(Jacq, 2001)

Dans les facultés des sciences, les TP doivent être effectués en petits groupes d'étudiants, pour permettre de concrétiser la théorie apprise en cours via des expériences. Les TP stimulent la curiosité des étudiants en leur permettant d'observer et de se poser des questions. Ils permettent aussi de développer un esprit d'initiative et surtout l'esprit critique quand il s'agit d'analyser et d'interpréter les résultats. En effet la démarche expérimentale aide d'une part à maitriser les concepts qui gèrent le fonctionnement d'un dispositif et d'autre part à articuler les pratiques expérimentales pour aboutir à une appropriation de connaissances qualifiées de théoriques. Cependant, les séances des TP dans les établissements marocains sont impactées par la massification. Au problème de l'accroissement de l'effectif des inscrits vient s'ajouter l'insuffisance des équipements scientifiques : matériels devant être surveillés tout au long des expériences afin d'éviter tout endommagement. Cette situation a généré certaines complexités de gestion, à la fois pour les enseignants et pour les étudiants. Au niveau des enseignants, le taux d'encadrement augmente au fur et à mesure que le nombre des inscrits augmente, on parle d'un effectif qui varie de trois jusqu'à quatre trinômes par enseignant, étant donné le volume horaire par séance réservé aux travaux pratiques, la répartition temporelle n'est guère suffisante pour couvrir et atteindre tous les objectifs avec efficacité.

5 Face à ces impératifs, les étudiants sont tenus désormais d'approfondir la compréhension des concepts théoriques et de s'approprier les savoir-faire techniques : maitriser les aspects théoriques liés à la manipulation, manier correctement le matériel, lire et interpréter les résultats et enfin extraire des conclusions constructives. Réaliser ces différents apprentissages et faire preuve de tels comportements ne peuvent aboutir entièrement et uniquement pendant les séances réservées aux travaux pratiques dans les conditions citées auparavant. Par ailleurs, on ne peut passer sous silence l'intérêt grandissant pour l'utilisation des Technologies de l'Information et de la 
Communication (TIC) en enseignement supérieur d'autant que les étudiants d'aujourd'hui pensent et traitent l'information d'une manière fondamentalement différente de leurs prédécesseurs.

6 La pédagogie inversée ou classe inversée nous semble être une solution pertinente pour répondre à cette problématique, c'est une pédagogie qui met l'apprenant au milieu du processus de l'enseignement/apprentissage comme acteur principal de sa formation et du développement de ses propres compétences. Dans cette lignée, la présente communication interroge donc le fait que la classe inversée puisse être un modèle adéquat pour instaurer une culture de réforme en travaux pratiques. Cette initiative a pour objectif de fournir un cadre de référence favorisant des modalités d'apprentissage actif et un enseignement davantage centré sur les apprenants dans les universités marocaines en vue de rendre fructueuse l'acquisition des apprentissages en travaux pratiques.

\section{Cadre théorique}

7 La classe inversée est une stratégie pédagogique qui réorganise l'espace/temps pour enseigner et apprendre (Lebrun, 2015), tout en s'appuyant sur les TIC. Elle fait appel à des méthodes pédagogiques bien connues en dehors de la classe et des méthodes actives centrées sur l'apprenant dans la classe. En effet, elle combine les caractéristiques de plusieurs approches pédagogiques parmi lesquelles on peut citer la pédagogie active, la différenciation pédagogique, l'auto-apprentissage, et l'apprentissage collaboratif. Elle s'appuie sur une démarche socioconstructiviste et mobilise de façon intensive les TIC tels que : forums, formulaire, exerciseurs, textes collaboratif, cartes mentales, ... (Guilbault et Viau-Guay, 2017). Par ailleurs, Marcel Lebrun, professeur à l'Université catholique de Louvain, présente trois types de classe inversée (Lebrun, 2016). Le Type 1 correspond au schéma "classique» de la classe inversée "Les leçons à la maison et les devoirs en classe». Cette pratique de la classe inversée a été baptisée par Eric Mazur, professeur de physique à Harvard, "qui demande à ses étudiants de lire son ouvrage de référence et ses notes de cours en amont pour consacrer ses enseignements aux difficultés exprimées par les étudiants, à des approfondissements et à différents exercices " (Lebrun et Lecoq, 2017). Eric Mazur publie ensuite en 1997 un ouvrage consacré à l'instruction par les pairs, associée à la pédagogie inversée (Mazur, 1997; Crouch et Mazur, 2001). Cela permet de faire remonter assez loin le concept de la "classe inversée ». Dans le Type 2 de la classe inversée relaté par Lebrun, les activités à distance sont effectuées par les élèves euxmêmes, de manière autonome ou en groupe, en amont de l'activité en classe. Cette deuxième pratique rejoint le même principe appelé «Classes renversées » (Cailliez et Henin, 2017) à travers lequel les élèves manipulent eux-mêmes les connaissances récoltées sur Internet hors classe, à la fois comme didacticiens et ingénieurs pédagogiques pour préparer et présenter des activités à leurs collègues en classe. Le Type 3 représente un mélange de classes inversées réunissant les deux premiers types dans des proportions diverses.

8 Nous retenons pour la suite comme définition de la classe inversée celle du service de soutien à la formation de l'Université de Sherbrooke: «les contenus de cours sont livrés au moyen de ressources consultables en ligne - le plus souvent des capsules vidéo - et le temps de classe est exclusivement consacré à des projets d'équipe, à des 
échanges avec l'enseignant et entre pairs, à des exercices pratiques et autres activités de collaboration » (Université de Sherbrooke, 2011).

9 A cet effet, la classe devient un lieu où les apprenants confrontent et soulèvent leur compréhension de la matière, grâce à des exercices d'apprentissage actif, principalement réalisés en groupe. Pour ce faire, ils se préparent avant le cours en faisant, par exemple, des lectures, ou en écoutant des vidéos, sur la matière qui sera travaillée en classe (Lebrun et Lecoq, 2017). L'enseignant, profitant du fait que les apprenants se sont déjà documentés en dehors de la classe, peut consacrer plus de temps à l'application, à la guidance individualisée, à la personnalisation et à la contextualisation (Lebrun, 2015). Les ressources à consulter hors classe en autonomie varient considérablement selon la place qu'elles occupent chronologiquement au sein de la séquence d'apprentissage (Dufour, 2014). A titre d'exemple, les concepts théoriques du cours peuvent s'insérer en début de séquence ou en milieu de séquence pour approfondir ou bien pour formaliser des notions approchées. Il peut aussi s'agir de la démonstration et de l'explication d'un dispositif technique ou d'une manipulation pratique qui sera réalisée en classe ce qui permet aux apprenants d'être plus autonomes lors de la mise en pratique (Nizet et Meyer, 2015). Selon des chercheurs qui ont pris du recul afin d'examiner les impacts possibles de cette réorganisation des activités pédagogiques (Bishop et Verleger, 2013 ; Karabulut-Ilgu, Jaramillo Cherrez et Jahren, 2017), les avantages à l'utilisation de la vidéo en enseignement peuvent se présenter comme suivant :

- Autonomie, contrôle accrus et possibilité de progression individualisée aux étudiants ;

- Accès à de nombreux contenus provenant de différentes sources ;

- Dialogue et échanges facilités entre les étudiants, les enseignants et des experts externes.

\section{Application de l'approche}

Nous proposons dans ce cadre, de faire recours à l'utilisation de la classe inversée pour la réalisation des travaux pratiques. Notre proposition consiste à renforcer la préparation préalable des manipulations en dehors des séances en présentiel. Les éléments clés visés sont :

- Maitriser les concepts liés aux incertitudes de mesure ;

- Identifier les risques liés aux montages des circuits électroniques ;

- Utiliser correctement les instruments de mesure en concret dans les différentes expériences pratiques ;

- Savoir rédiger correctement un compte-rendu de TP ;

- Réaliser à travers des études de cas les mesures électroniques de base.

11 Pour ce faire, nous avons mis en ligne une formation asynchrone qui met à la disposition des étudiants deux catégories de vidéos: la première cible l'aide à l'apprentissage concernant les dispositifs communs aux différentes expériences programmées. Ces vidéos s'intéressent en particulier à l'explication de l'usage des appareils électroniques intervenant aux TP, y compris les techniques de mesure. La deuxième catégorie de vidéos se focalise sur les particularités de chaque expérience, à savoir le montage complet et la mise en marche de la manipulation en question. Des exercices par simulation sont notamment proposés pour apprécier l'évolution de l'apprentissage chez les étudiants via un espace d'interaction graphique et animé. 
12 L'évaluation-test de notre approche est effectuée dans un premier temps pour les travaux pratiques de « l'électromagnétisme dans le vide " pour le compte des étudiants inscrits en troisième semestre, TP communs en général à différentes filières des facultés des sciences marocaines.

Les objectifs visés par cette autoformation portent sur les trois points suivants :

- Connaissances et prérequis : les étudiants sont invités à revoir des concepts scientifiques de base, définitions, unités, modèles, ordres de grandeur, exemples d'application....

- Méthodologie de travail : elle concerne l'utilisation raisonnée des lois et formules, de calcul des incertitudes, les exploitations de courbes, des méthodes de raisonnement.

- Méthodologie expérimentale : elle cible le savoir-faire pratique concernant les risques du maniement des dispositifs électriques, l'utilisation des appareils de mesure, la traduction graphique des mesures et leur exploitation.

\section{Expérimentation}

Cette étude s'est déroulée à l'Université Marocaine Chouaib Doukkali et plus particulièrement à la faculté des sciences dont l'une des missions est d'assurer la mise en place des travaux pratiques (TP). Dans ce contexte, nous comptons contribuer à améliorer et faciliter la réalisation de cette mission pour les TP de Physique qui constitueront pour nous le domaine d'apprentissage objet de la pédagogie inversée.

L'objectif de cette expérimentation est la mise en place de solutions fondamentales pour les problèmes rencontrés pendant les séances des TP de «l'électromagnétisme dans le vide » et ce pour la deuxième année de l'ensemble des filières du cycle LMD de la Faculté des Sciences. Pour ce faire, nous avons mis en ligne une autoformation via notre plateforme "CloudSpoc» (El Khadiri, Labouidya, Elkamoun et Hilal, 2017). Nous proposons de renforcer les connaissances théoriques liées à la pratique et instaurer une méthodologie de travail chez l'étudiant à travers des vidéos et des présentations pour pouvoir aboutir à une séance de TP fructueuse avec une bonne préparation, une utilisation correcte et sans danger des appareils et instruments de mesure et enfin une exploitation rigoureuse des résultats.

\subsection{Public cible}

16 Notre première expérimentation a été réalisée auprès d'étudiants inscrits au semestre 3 de la filière sciences mathématiques appliqués (SMA3) de la faculté des sciences d'El Jadida, qui ont suivi les travaux pratiques du module « l'électromagnétisme dans le vide » durant le session d'automne de l'année 2017. Les 53 étudiants concernés ont été appelés à suivre en amont la formation en ligne concernant l'appui à la préparation aux TP.

\subsection{Recueil des données}

Plusieurs techniques sont dédiées au recueil de données telles que l'entretien, le questionnaire et l'observation. Pour évaluer notre approche basée sur la classe inversée, nous avons fait appel à ces techniques en fonction du moment d'utilisation de 
celles-ci dans les processus d'enseignement/apprentissage (lors ou en dehors du déroulement).

L'assimilation des connaissances fournies par l'autoformation préparatoire en ligne est contrôlée par l'intermédiaire de questionnaires, à la fois en ligne via des QCM d'application concrète et en classe à travers des questions directes, liés uniquement au contenu de la formation dispensée pour pouvoir évaluer l'apport de notre approche. Pour estimer le niveau de connaissances des étudiants par rapport aux compétences requises aux travaux pratiques de "l'électromagnétisme dans le vide", nous avons fait appel à une évaluation sommative à travers l'examen de TP effectué en fin de session pour le public cible de la promotion 2017. Les résultats obtenus ont été comparés à ceux de la promotion précédente.

19 L'utilisation de plusieurs méthodes complémentaires rend l'analyse et l'explication des résultats plus pertinentes; nous avons tenté dans ce sens d'effectuer des entretiens individuels et de groupe avec notre public cible. Nous avons également fait appel à la technique d'observation durant les séances des travaux pratiques, pour contrôler la progression et le déroulement de l'apprentissage d'une part et évaluer l'acquisition réalisée d'autre part. Pour déceler de manière détaillée les difficultés qui ont pu survenir durant cette expérimentation, nous avons fait appel à la rétroaction des étudiants à cet apprentissage actif basé sur la classe inversée. A la fin de cet apprentissage et à l'aide d'un formulaire à questions fermées, les étudiants ont évalué ce processus d'apprentissage en exprimant leurs perceptions de manière anonyme. Les énoncés portaient sur trois catégories : Organisation, Impact et Implication.

\subsection{Organisation de l'expérimentation}

L'organigramme organisationnel de la formation préparatoire aux travaux pratiques proposée via la plateforme CloudSpoc (accessible en ligne à travers le lien http:// cloudspoc.ma), est présenté par la figure suivante : 
Figure 1. Organigramme organisationnel de la formation

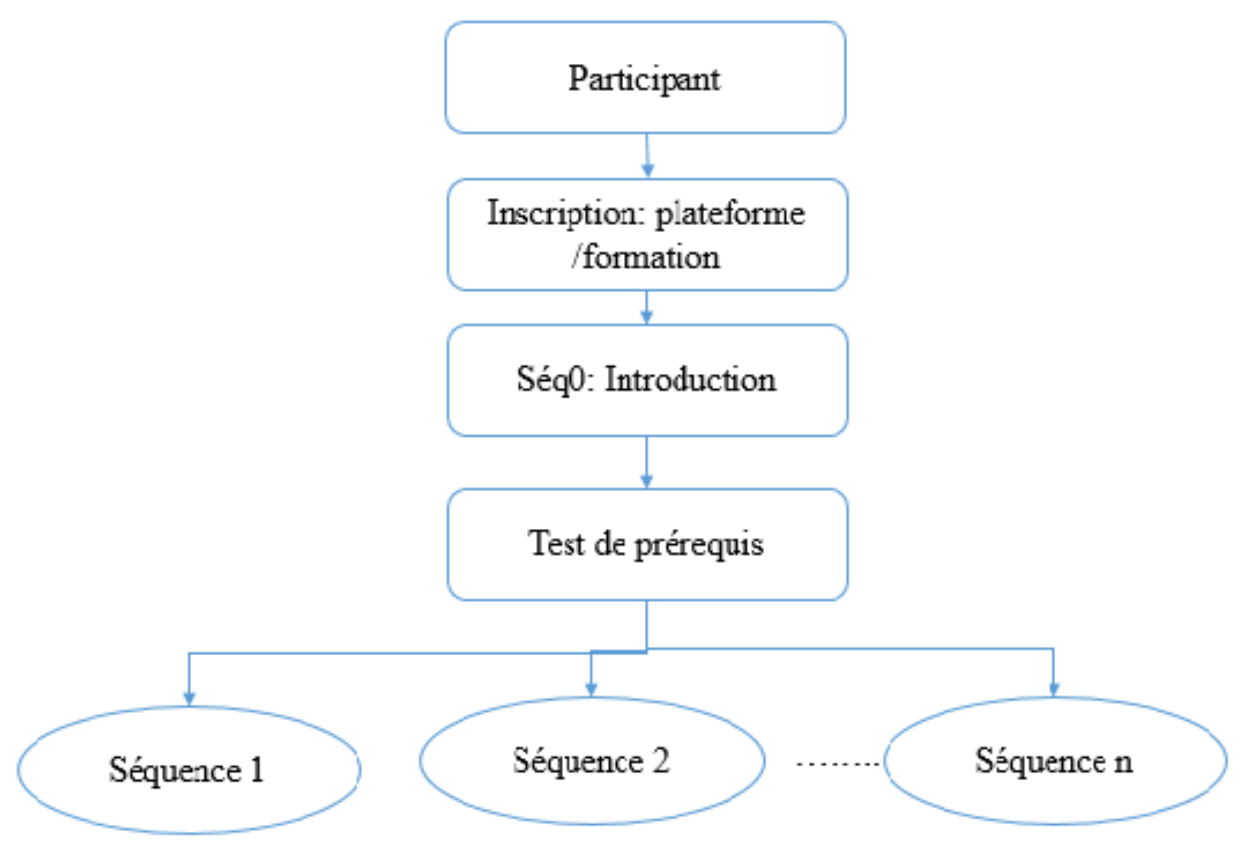

L'étudiant doit s'inscrire à la plateforme et disposer d'un login et mot de passe. L'inscription à la plateforme doit être suivie par une inscription à l'autoformation pour la préparation des TP d'électromagnétisme pour S3. En accédant à la plateforme, l'étudiant trouvera un guide d'utilisation des séquences sous forme d'une introduction. Nous avons choisi une démarche pédagogique qui consiste à varier les supports de formation proposés, en adoptant une approche d'apprentissage progressif dont le but est de permettre à l'étudiant de localiser dans un premier temps ses besoins en matière de formation via un Test d'entrée sur les prérequis. Ceci va lui permettre de cerner ses lacunes afin de pouvoir développer ses connaissances tout au long de la formation grâce à différentes ressources telles que les vidéos, les ressources complémentaires, les Quizz, ... Les étudiants ont été initiés à l'utilisation de la plateforme en présentiel avant le début de cette formation préparatoire. Lors de cette séance d'initiation, le rôle et l'objectif de chaque entité ont été bien expliqués tout en signalant qu'une note sera attribuée à cet apprentissage.

Le déroulement de cette autoformation est présenté sur la figure suivante : 
Figure 2. Séquencement et contenu de la formation en ligne

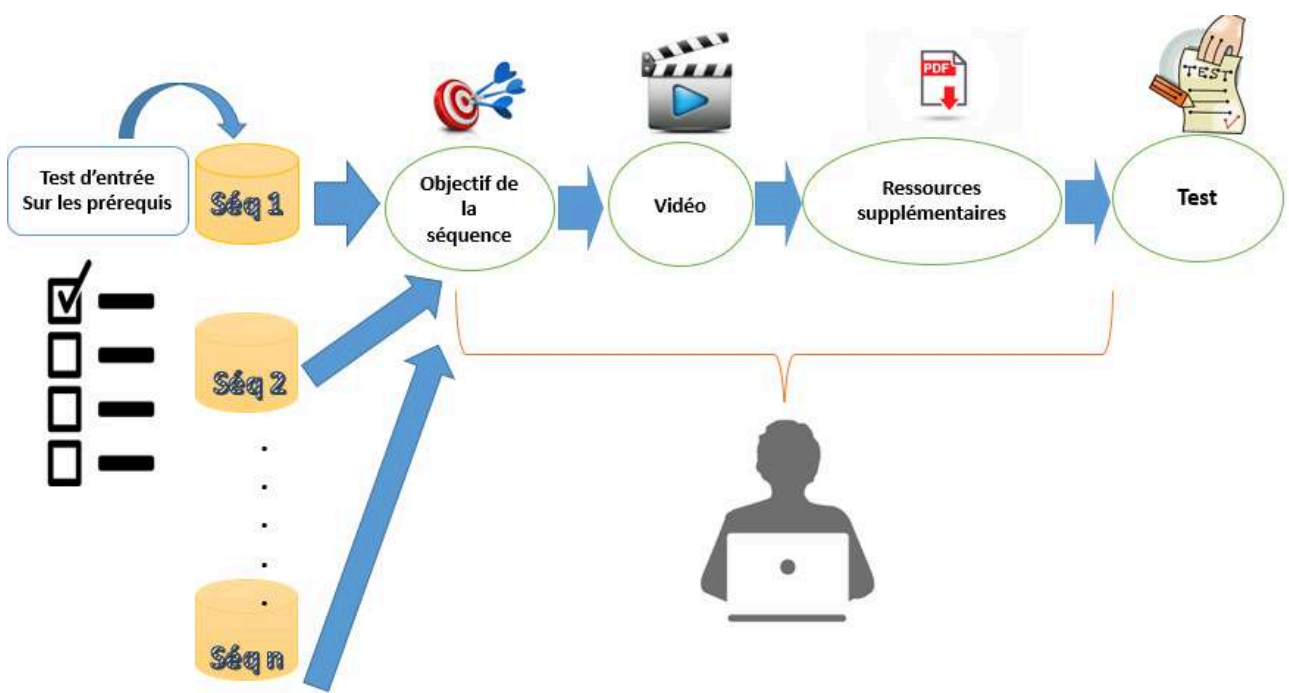

Les différentes séquences introductives aux travaux pratiques ont pour objectif de présenter la matière sous une autre forme que celle utilisée lors des cours en amphithéâtre ou lors des séances de travaux dirigés en classe. Nous faisons appel à des vidéos qui expliquent le fonctionnement des différents dispositifs électronique impliqués dans les manipulations, des vidéos pour les appareils de mesure et des vidéos pour le montage et réalisation de la partie expérimentale. En effet et pour chaque séquence, il s'agit de présenter dans un premier lieu l'ensemble des rappels théoriques concernant les notions liées aux travaux pratiques en question. Ensuite, une ou plusieurs vidéos récapitulant le contexte réel des notions présentées en entrée. Nous avons opté pour des séquences vidéo courtes (5 à 10 minutes) ayant pour but de rappeler et de mettre en application les concepts théoriques de base qui seront utilisés pendant les séances des TP.

Nous présentons sur les figures suivantes une revue de la disposition de quelques supports selon un exemple de parcours donné :

Figure 3. Présentation vidéo de la séquence

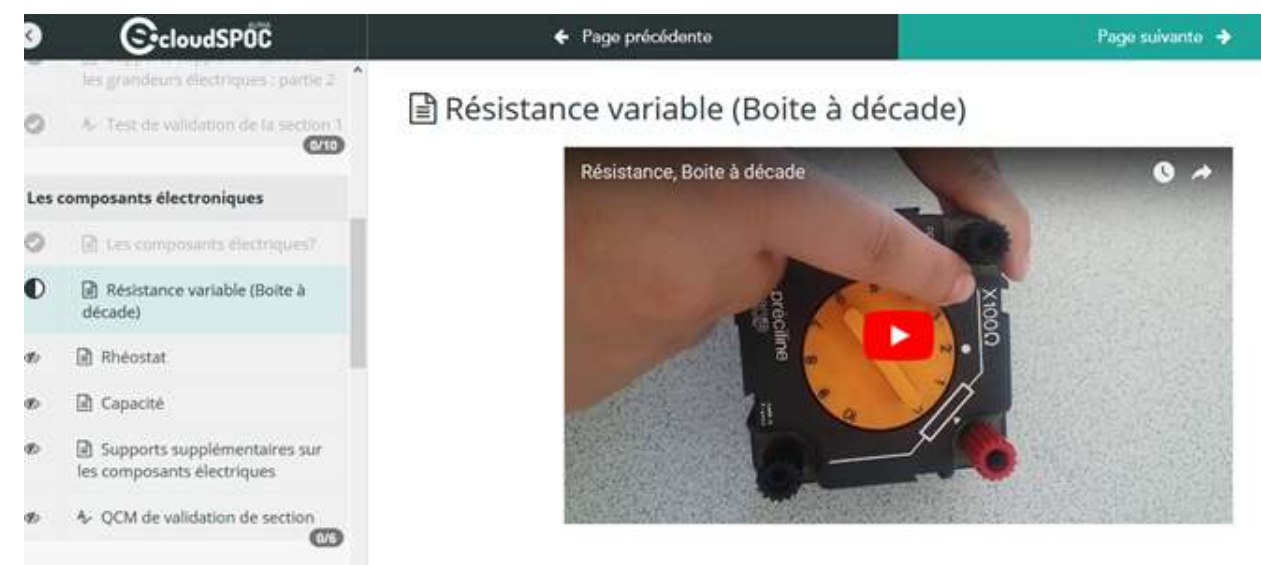


Figure 4. Présentation Ressources complémentaire de la séquence sous format pdf

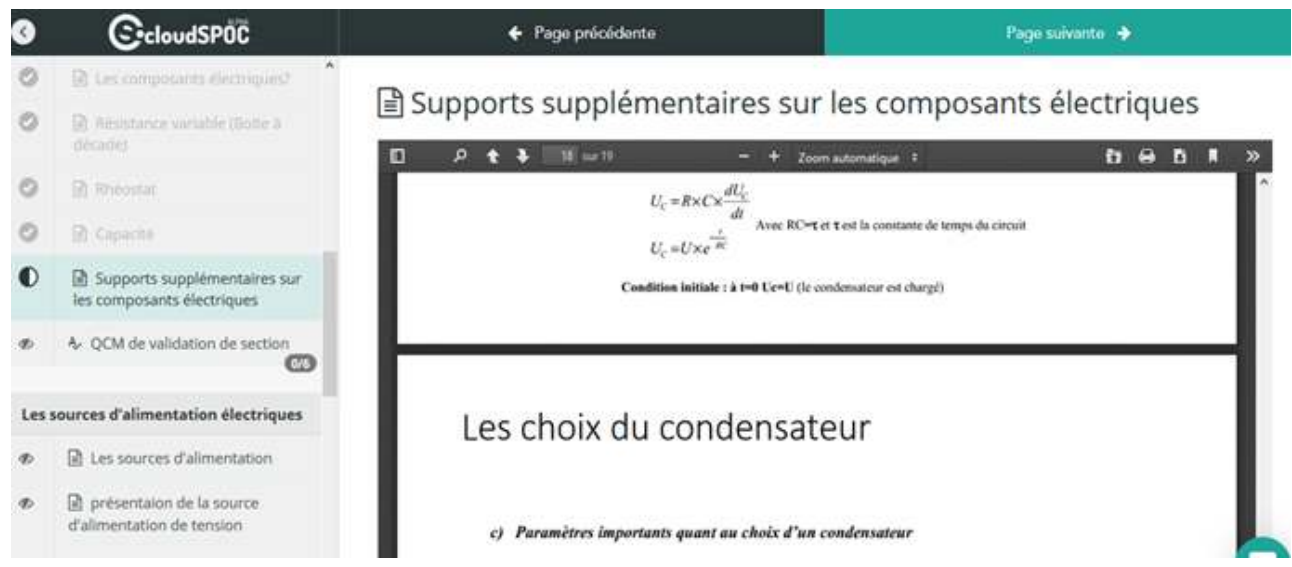

Figure 5. QCM de validation de la séquence

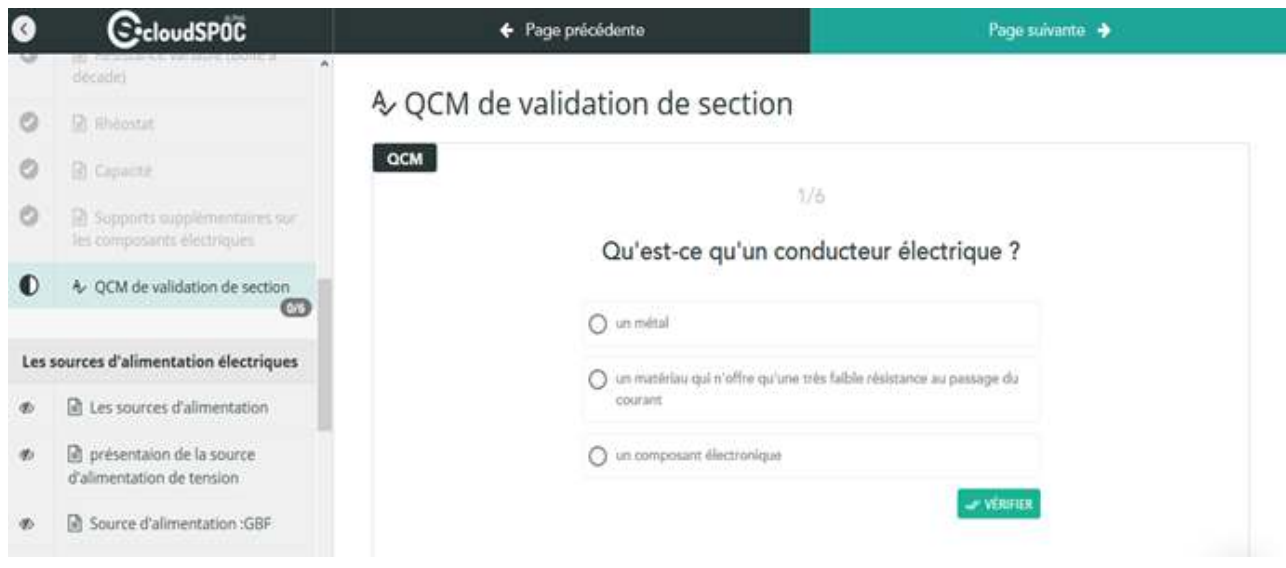

\subsection{Résultats de l'évaluation de notre approche}

25 L'évaluation d'un dispositif d'enseignement quelle qu'en soit la nature n'est pas une tâche simple. Il s'agit d'un processus complexe étroitement lié à plusieurs statistiques décisionnelles qui ne sont pas forcément quantifiables. D'ailleurs, l'évaluation ne doit pas se confiner seulement aux résultats empiriques mais doit aussi comprendre les résultats d'interactions entre les différentes entités impliquées dans l'expérimentation. Pour ce faire, nous avons pris en considération pour l'évaluation de notre approche l'ensemble des résultats suivants :

- Le Pré-Test et le Post-Test

- L'évaluation sommative

- Les entretiens individuels et de groupe

- L'observation des enseignants

- La rétroaction des étudiants

\subsubsection{Résultats des Pré-Test et Post-Test}

Nous avons utilisé le même questionnaire au format papier pour le Pré-Test et le PostTest. Ce questionnaire est constitué d'items courts et directs à réponse unique. Ce type de questions permet d'une part, d'impliquer les étudiants et de les inciter à suivre 
l'autoformation dispensée en ligne et d'autre part, il va nous faciliter par la suite la catégorisation des réponses. Le Pré-Test est effectué par les étudiants tout au début de la séance de TP en présentiel et le Post-Test à la fin de celle-ci.

Figure 6. Résultats du Pré-Test et du Post-Test

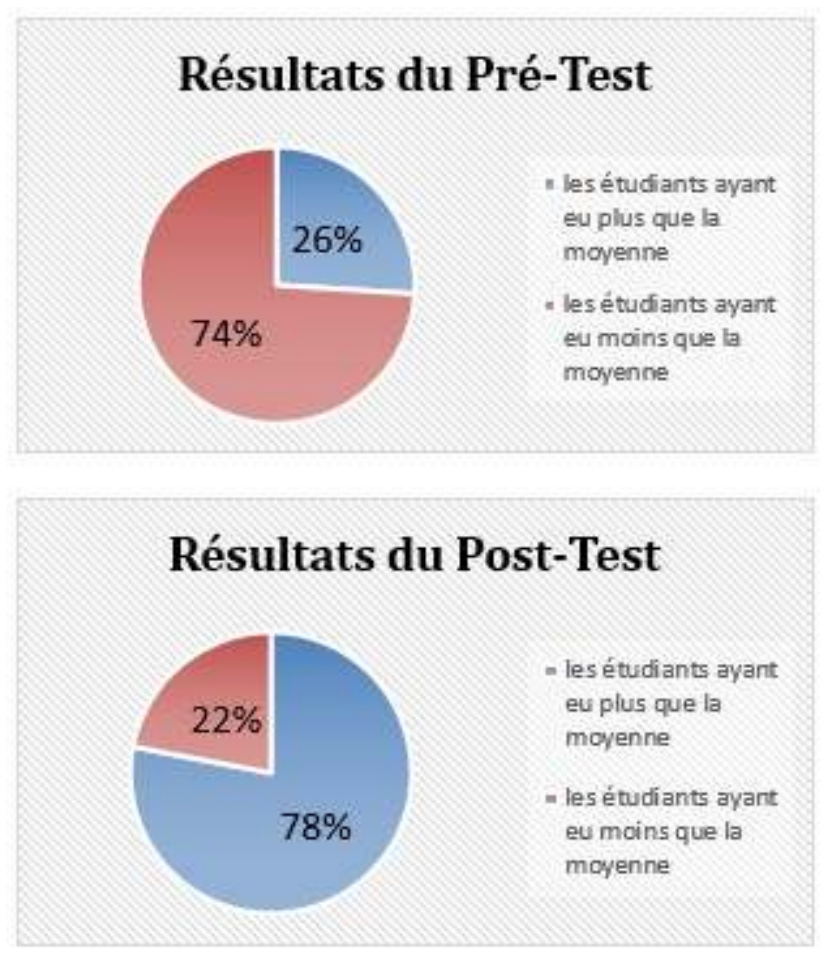

Nous tenons à signaler d'abord que même si tous les étudiants ont confirmé leur inscription au dispositif, les statistiques données par la plateforme ont confirmé que, sur l'échantillon de 53 étudiants, seulement 49 d'entre eux se sont effectivement inscrits à la plateforme et ont suivi la formation en ligne.

En examinant les résultats du Pré-Test, nous avons remarqué que seulement $26 \%$ des étudiants parmi les 49 ont suivi la formation en ligne (hors classe avant la séance de $\mathrm{TP})$, ont pu répondre correctement aux questions et ont pu avoir une note supérieure ou égale à la moyenne (Moyenne $=10 / 20$ ). Il est à noter que l'échantillon des étudiants examiné contient un certain nombre d'étudiants qui ont refait ces TP suite à une nonvalidation antérieure du module concerné. En ce qui concerne les résultats du Post-Test (après la séance de TP en classe), on remarque que $78 \%$ des étudiants parmi les 53 ont réussi à répondre correctement aux questions.

Les résultats du Post-Test montrent que l'amélioration du niveau de connaissance des étudiants est apparue après la séance de TP en classe suite à l'accompagnement et le suivi des enseignants encadrants. Les résultats du Pré-Test sont en concordance avec ceux de la figure 8 ci-après qui indique que seulement $32,7 \%$ des étudiants ont pu suivre la formation jusqu'au bout. Ces résultats montrent d'une part que nos étudiants ne sont pas encore habitués à ce type d'apprentissage en autoformation en ligne car notre expérimentation est la première du genre pour eux. D'autres part, un bon nombre d'étudiants étaient réticents à la passation du test de positionnement au début de cette formation de peur qu'il soit pris en considération dans la note finale des TP (voir partie: Résultats des entretiens). Il ne faut pas aussi négliger le manque 
d'implication de certains étudiants, si on combine les résultats des figures 7 et 10 : à peu près $66 \%$ des étudiants ont sollicité la formation plutôt et seulement le weekend (figure 7) et $69 \%$ d'entre eux ont consacré moins d'une heure par jour à cette formation [figure 10]).

\subsubsection{Résultats de l'évaluation sommative}

Pour estimer le niveau de connaissances des étudiants par rapport aux compétences requises aux travaux pratiques de "l'électromagnétisme dans le vide ", nous avons fait appel à une évaluation sommative à travers l'examen de TP effectué en fin de session pour le public cible de la promotion 2017. Les résultats obtenus ont été comparés à ceux de la promotion précédente (groupe témoin) concernant la même filière. Pour mener à bien cette comparaison, nous avons veillé à assurer un même degré de difficulté concernant l'examen du TP par rapport aux deux promotions. La figure suivante illustre le pourcentage de l'effectif des étudiants classés en trois catégories: les étudiants qui n'ont pas réussi à avoir la moyenne en TP (Note $<10)$, les étudiants ayant une note insuffisante pour être dispensé des TP en cas de non-validation du module concerné $(10 \leq$ Note $<12)$ et les étudiants ayant une note suffisante pour être dispensé des TP dans le même cas de non-validation du module concerné (Note $\geq 12$ ).

Figure 7. Comparaison des notes (en \%) de l'examen de TP pour les promotions 2016 et 2017

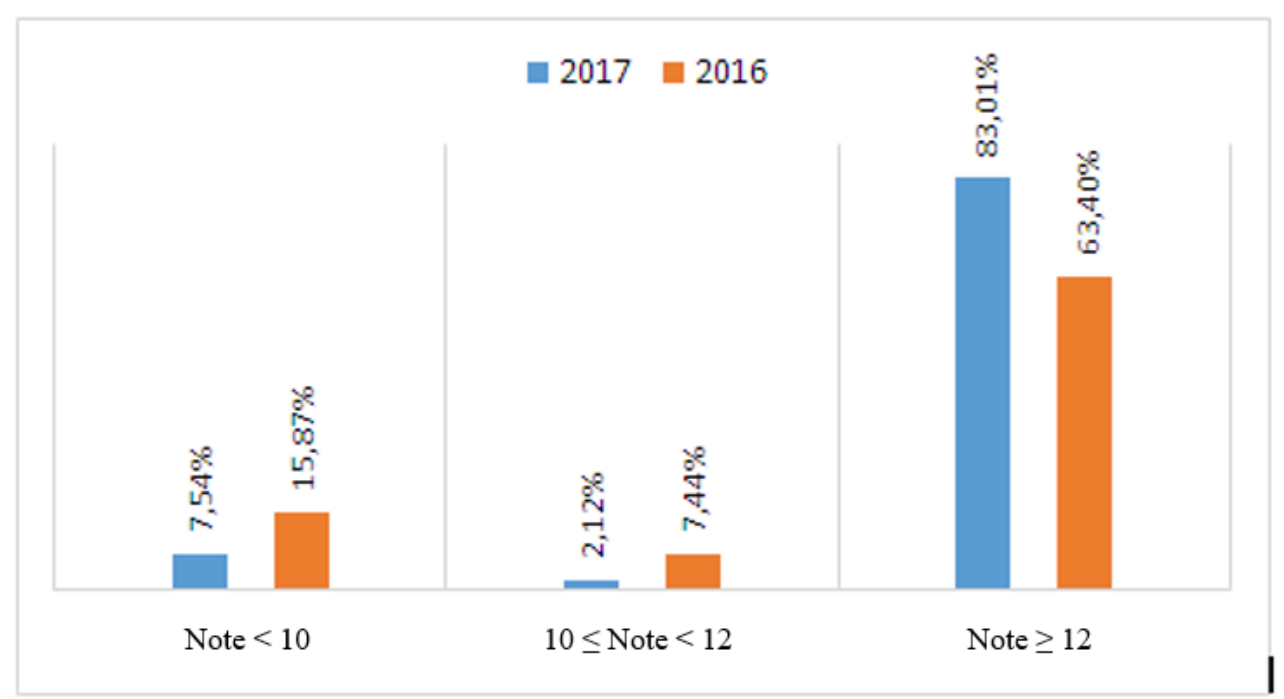

On peut observer une nette amélioration des notes pour les trois catégories considérées. Le nombre des étudiants qui n'ont pas réussi à obtenir la moyenne (10/20) pour l'examen du TP représente $15,87 \%$ pour la promotion 2016, alors qu'il a été réduit à $7,54 \%$ pour la promotion 2017 , promotion qui a bénéficié de l'autoformation de préparation préalable aux TP. Le nombre d'étudiants qui n'ont pas réussi à valider les TP en question a été réduit de $5 \%$. Le pourcentage des étudiants qui ont validé les TP avec une bonne note a augmenté de $20 \%$ pour la promotion 2017 par rapport à 2016 . Suite à ces résultats, on peut affirmer que l'autoformation de préparation aux TP dispensée aux étudiants de la promotion 2017 a été bénéfique pour eux puisqu'elle leur a permis une progression individualisée en consultant les vidéos en ligne quand ils le veulent, où ils le veulent et autant de fois qu'ils le désirent. En particulier, ces vidéos mises à la disposition des étudiants permettent d'affiner la vérification des acquis, 
d'accéder à différentes aides techniques ou théoriques ce qui rend possible une mise à jour des connaissances et une révision de préparation à l'examen des TP indépendamment de l'enseignant encadrant.

\subsubsection{Résultats des entretiens individuels et de groupe} été menés pendant les séances des TP. Ces entretiens ont pour objectif d'évaluer notre dispositif d'autoformation de préparation aux TP à travers les perceptions des étudiants dans un environnement social. En terme d'efficacité, les étudiants ont bien évidement confirmé que les sources d'information disponibles sur la plateforme, leur ont permis de comprendre plusieurs nouvelles notions, surtout celles qui expliquent le déroulement de la manipulation et l'utilisation des dispositifs électriques et de mesure. La majorité des étudiants a exprimé sa satisfaction du dispositif et a affirmé que l'idée était nouvelle et intéressante dans la mesure où elle lui a permis de bien se préparer.

Au niveau de l'acceptabilité, $80 \%$ des étudiants ont confirmé qu'ils étaient motivés pour suivre cette formation et pour découvrir pour la première fois cette nouvelle forme de l'apprentissage autonome en ligne. Cependant un certain nombre d'étudiants ( $20 \%)$ a avoué avoir été freiné par le Test d'entrée sur les prérequis de peur qu'il soit pris en considération dans l'évaluation des apprentissages pour les TP en question. Il est à noter, comme on l'avait expliqué aux étudiants durant la séance d'initiation en présentiel, que ce test de positionnement a été mis en place pour permettre aux étudiants de tester leur prérequis mais qui n'empêchait en aucun cas l'accès aux différentes ressources disponibles. Toujours concernant l'évaluation des TP, quelques étudiant ont demandé encore une fois si une note serait attribuée à cette autoformation à travers les divers tests et si elle serait prise en compte dans la note globale des TP en question, chose qui a été aussi signalée par les encadrants lors de la séance d'initiation. En conclusion de cette partie, nous constatons que l'évaluation notée reste un sujet de préoccupation récurrent parmi les étudiants plus que l'acquisition des apprentissages. Par ailleurs, les étudiants en difficulté ont exprimé qu'ils ont rencontré certains obstacles qui ont contrarié le bon suivi de la formation, tel que de la charge horaire et aussi le handicap de la langue.

\subsubsection{Résultats de l'observation des enseignants}

Nous avons effectué un suivi attentif des étudiants au cours des différentes séances de travaux pratiques afin de déceler des changements d'attitude éventuels chez nos étudiants suite à l'autoformation en ligne dédiée à la préparation préalable des TP. Nous rappelons ici que l'objectif fondamental de cette initiative est de rendre fructueuse une séance de TP étant donné que les étudiants auraient bien préparés les TP hors classe, ce qui leur permettraient d'être plus autonomes lors de la mise en pratique en classe et l'enseignant encadrant pourrait ainsi consacrer plus de temps à la guidance individualisée, à la personnalisation et à la contextualisation. Suite à nos observations, il s'est avéré que certaines équipes se sont bien débrouillées et sollicitaient rarement l'intervention des enseignants encadrants et ce pratiquement tout au long des séances de TP. Nous avons vérifié et noté que ces étudiants sont des nouveaux inscrits c'est-à-dire qu'ils n'ont jamais fait les TP en question. Ces éléments constituent $34 \%$ de l'effectif des étudiants par groupe de TP.

Revue internationale de pédagogie de l'enseignement supérieur, 34(3) | 2018 


\subsubsection{Résultats de la rétroaction des étudiants}

35 A la fin de cet apprentissage, les étudiants ont évalué ce processus d'apprentissage en exprimant leurs avis de manière anonyme selon trois catégories de questions: Organisation, Impact et Implication.

Figure 8. Moment de la semaine pendant lequel les étudiants ont sollicité l'autoformation asynchrone en ligne

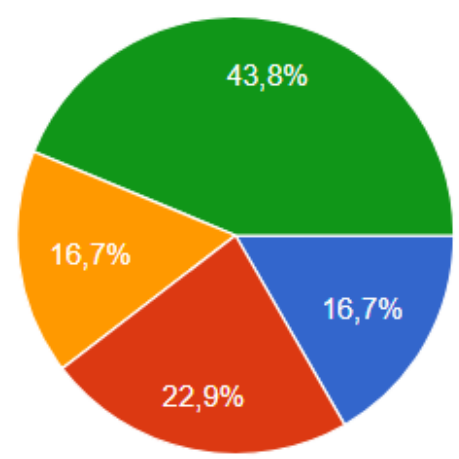

Surtout durant la semaine, peu le week-end

Surtout durant le week-end, peu en semaine

En semaine ou le week-end à égalité

Seulement le week-end

La figure 8 montre que $65,8 \%$ des étudiants affirment avoir travaillé sur le dispositif en grande partie pendent le weekend. Ceci est dû principalement à la charge horaire des enseignements, d'où ils ont déclaré qu'ils n'ont pas eu le temps nécessaire pour faire les préparations comme il se doit en dehors des weekends.

Figure 9. Pourcentage de réalisation de la formation préparatoire aux TP proposés

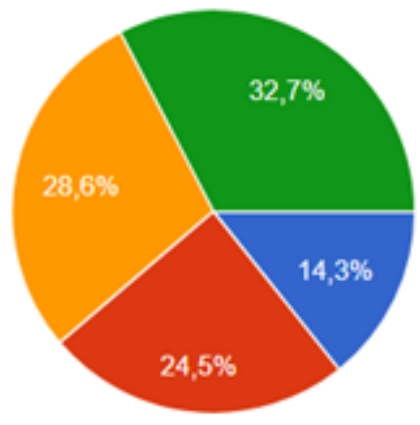

Sur la figure 9 , nous constatons que seulement $32,7 \%$ des étudiants ont pu suivre la formation jusqu'au bout au moment où $28,6 \%$ ont atteint $75 \%$. Les deux chiffres correspondent donc à l'effectif des étudiants qui ont pu manifester une vraie autonomie lors du déroulement des séances des travaux pratiques. 
Figure 10. Le moyen de connexion internet utilisé par les étudiants lors de l'accès à la plateforme

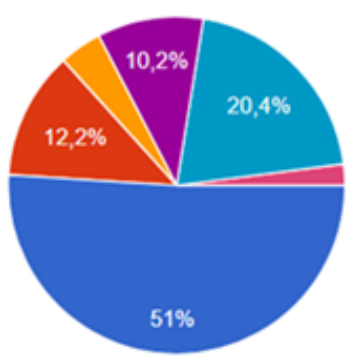

Connexion $4 \mathrm{G}$

Connexion wifi ou par cable dans mon

établissement de formation

Nous n'avons pas intéragi

Connexion wifi dans un établissement public

Dans un cybercafé

- Autre type de connexion

Sur la figure 11, nous remarquons que $49 \%$ ont affirmé avoir consacré $1 / 2$ heure à une heure par jour pour la formation. En se référant aux résultats où ils ont confirmé avoir travaillé en grande partie pendant le weekend, nous pouvons conclure que le temps alloué n'était pas assez suffisant pour mener à bien la préparation des TP hors classe et passer les séances consacrées aux TP en présentiel dans de bonnes conditions. Vu que ces étudiants demeurent incapable de travailler correctement et de manière automne, nous pensons faire appel à des planifications d'accès et un suivi de la progression des apprentissage en tenant compte de la possibilité d'imposer une remédiation pour les étudiants en difficulté au niveau de la plateforme.

41 Nous pouvons conclure que les résultats de la réussite du dispositif dépendent en grande partie du degré d'implication des étudiants. Un facteur relatif qui est aussi

Revue internationale de pédagogie de l'enseignement supérieur, 34(3) | 2018 
impacté par d'autres conditions: charge du volume horaire connectivité internet, motivation, ...

\section{Conclusion et perspectives}

Les chercheurs tentent toujours d'apporter des réponses à certaines questions comme celle de l'efficacité et de la pertinence de l'usage des TIC pour l'enseignement / apprentissage. Ils ont cherché pour longtemps ces réponses dans un contexte précis qui permet de les valider. Dans notre cas, nous avons essayé d'apporter à la fois une contribution et aussi une réponse à l'efficacité de l'usage des TIC dans l'enseignement supérieur, via le déploiement des classes inversées outillée pour la préparation préalable aux travaux pratiques de physique. Notre proposition d'utiliser les classes inversées comme un outil innovateur permettant de résoudre un certain nombre de problèmes liés à l'aboutissement des travaux pratiques dans l'université.

En effet, et pour une première expérimentation, les résultats obtenus sont très encourageants, nous avons remarqué qu'au niveau de l'acceptabilité, $80 \%$ des étudiants ont confirmé qu'ils étaient motivés pour suivre cette formation et pour découvrir pour la première fois cette nouvelle forme de l'apprentissage autonome en ligne. De point de vue efficacité, on peut affirmer que l'autoformation de préparation aux TP dispensée aux étudiants de la promotion 2017 a été bénéfique pour eux puisqu'elle leur a permis d'affiner la vérification des acquis, d'accéder à différentes aides techniques ou théoriques ce qui rend possible une mise à jour des connaissances et une révision de préparation à l'examen des TP indépendamment de l'enseignant encadrant.

Par ailleurs, nous avons pu déceler un certain nombre de contraintes auxquelles il faut faire face pour améliorer notre dispositif, que ça soit d'ordre technique ou humain. Les différents résultats obtenus nous ont aussi permis de comprendre les pratiques et fonctionnalités actuelles de notre approche afin de faire la lumière sur les mises en œuvre futures.

\section{BIBLIOGRAPHIE}

Abrahams, I. et Millar, R. (2008). Does practical work really work? A study of the effectiveness of practical work as a teaching and learning method in school science. International Journal of Science Education, 30(14), 1945-1969.

Abrahams, I., Reiss, M.J. et Sharpe, R.M. (2013). The assessment of practical work in school science. Studies in Science Education, 49(2), 209-251.

Bishop, J.L. et Verleger, M. (2013). The Flipped Classroom: A Survey of the Research. Dans 120TH ASEE Annual Conference \& Exposition. Paper ID 6219. Repéré à https://www.asee.org/public/ conferences/20/papers/6219/view.

Cailliez, J.-C., et Henin C. (2017). La classe renversée - L'Innovation pédagogique par le changement de posture. Paris: Ellipses Marketing. 
Crouch C. H. et Mazur E. (2001). Peer Instruction: Ten years of experience and results. American Journal of Physics, 69(9), 970-977. DOI: 10.1119/1.1374249. Repéré à http://web.mit.edu/jbelcher/ www/TEALref/Crouch_Mazur.pdf.

Dillon, J. (2008). A review of the research on practical work in school science. London : King's College.

Dufour, H. (2014). La classe inversée. Technologie. Repéré à http://eduscol.education.fr/sti/sites/ eduscol.education.fr.sti/files/ressources/techniques/6508/6508-193-p44.pdf.

El Khadiri, K., Labouidya, O., Elkamoun, N. et Hilal, R. (2017). CloudSPOC: Additional face-to-face courses for an evolving higher education. IJCSNS, 17(8), 150. Repéré à : http://paper.ijcsns.org/ 07_book/201708/20170820.pdf.

Guilbault, M. et Viau-Guay, A. (2017). La classe inversée comme approche pédagogique en enseignement supérieur : état des connaissances scientifiques et recommandations. Revue internationale de pédagogie de l'enseignement supérieur, 33(1). Repéré à http://ripes.revues.org/1193.

Hofstein, A. (2004). The laboratory in chemistry education: Thirty years of experience with developments, implementation, and research. Chemistry education research and practice, 5(3), 247-264.

Jacq, J. (2001). Des TP : Pourquoi ?. Réflexion sur les travaux pratiques en Sciences physiques et chimiques fondamentales et appliquées. Repéré à www.spc.ac-aix-marseille.fr/phy_chi/Info/TP/ Des_TP_Pourquoi.doc.

Karabulut-Ilgu, A., Jaramillo Cherrez, N. et Jahren, C.T. (2018). A systematic review of research on the flipped learning method in engineering education. British Journal of Educational Technology, 49(3), 398-411. doi: 0.1111/bjet.12548.

Lebrun M., (2015). Classe inversée? Oui, mais... quoi et comment ? Repéré à http:// enseignement.catholique.be/segec/fileadmin/DocsFede/Service_segec/etude/traces_2015/ Transcription_\%20Lebrun.PDF.

Lebrun M., (2016). La pédagogie inversée : Enseigner autrement dans le supérieur avec la classe inversée. De Boeck Supérieur. ISBN 978-2-8073-0618-9.

Lebrun M., et Lecoq J. (2017). Guide pratique pour débuter en classe inversée (inspiré de leur ouvrage «Classes inversées. Enseigner et apprendre à l'endroit » paru en 2015 aux éditions Canopé). En ligne : https://cdn.uclouvain.be/groups/cms-editors-lll/carnets/Classes_Inversees.pdf.

Lunetta, V.N., Hofstein, A. et Clough, M.P., (2007). Learning and teaching in the school science laboratory: An analysis of research, theory, and practice. Dans Handbook of research on science education (p. 393-441).

Mazur, E (1997). Peer Instruction: A User's Manual. Physics Today, 50(4), 65. Repéré à https:// doi.org/10.1063/1.881735.

Morin, E. (1984). Dialogue sur le sujet qui écrit. Cahiers Pierre-Baptiste, 13, 47-62.

Nizet, I. et Meyer, F. (2015). La classe inversée : que peut-elle apporter aux enseignants. Répéré à http://www.cndp.fr/agence-usages-tice/que-dit-la-recherche/la-classe-inversee-que-peut-elleapporter-aux-enseignants-79.htm.

Pekmez, E.S., Johnson, P. et Gott, R. (2005). Teachers' understanding of the nature and purpose of practical work. Research in Science \& Technological Education, 23(1), 3-23.

Prost, A., (1986). L'enseignement s'est-il démocratisé ? Les élèves des lycées et collèges de l'agglomération d'Orléans de 1945 à 1980. Paris : Presses universitaires de France. 
Université de Sherbrooke (2011). Le SSF veille. Faire la classe mais à l'envers : la flipped classroom. Sherbrooke : Université de Sherbrooke, Service de soutien à la formation. Repéré à https:// www.usherbrooke.ca/ssf/veille/perspectives-ssf/numeros-precedents/novembre-2011/le-ssfveille/faire-la-classe-mais-a-lenvers-la-flipped-classroom.

Vétois, J. (2016). Les nouvelles technologies dans l'enseignement et l'apprentissage. Besoins, utilisations et rentabilités. Terminal. Technologie de l'information, culture \& société, (118). Repéré à https://journals.openedition.org/terminal/1401.

Vindevoghel M. et Blondeau J.M. (1998). Autoformation éducative et enseignement universitaire sur mesure. Dans Huitièmes Journées Informatique et Pédagogie des Sciences Physiques, IUFM de Montpellier. Repéré à http://www.inrp.fr/Tecne/Rencontre/Jipsp8/Pdf/AutoForm.pdf.

\section{RÉSUMÉS}

Le secteur de l'enseignement supérieur a beaucoup évolué ces dernières années, suite à un certain nombre de facteurs tels que la démocratisation, la massification d'accès, ainsi que l'évolution des pratiques d'apprentissage des étudiants issus de la culture numérique. Ces catalyseurs poussent les pratiques pédagogiques des enseignants à être questionnées et surtout à changer. Nous proposons dans ce cadre d'utiliser les classes inversées pour renforcer la préparation préalable des travaux pratiques. Pour ce faire, nous avons mis en place une autoformation en ligne basée sur une scénarisation pédagogique de l'apprentissage en question. Une première expérimentation de la démarche proposée s'est déroulée au cours de l'année universitaire 2017/18 concernant les travaux pratiques de "l'électromagnétisme dans le vide " pour le compte d'étudiants inscrits en semestre 3. Cette méthodologie met aussi l'accent sur la réciprocité du lien entre qualité et efficacité de la pratique pédagogique adoptée d'une part et apprentissages des étudiants d'une autre part à travers une enquête de satisfaction. L'analyse des différents résultats obtenus sont encourageants et renseignent sur les points à améliorer au niveau de notre dispositif.

In the last few years, the higher education sector has considerably evolved, following a number of factors such as democratization, access massification, as well as the evolution of learning practices of students due to the invasion of digital culture. These catalysts push the teaching practices of teachers to be questioned and specially to change. We propose in this context to use the flipped classrooms to reinforce the preliminary preparation of practical work. To do this, we have set up an online self-training based on an educational scenario of the learning in question. A first experimentation of the proposed approach took place during the academic year 2017/18 concerning the practical work of "electromagnetism in a vacuum" on behalf of students registrated in semester 3 . This methodology also introduces emphasis on the reciprocity of the link between quality and effectiveness of the pedagogical practice adopted on the one hand and learning of students on the other hand through a satisfaction survey. The analysis of the different results obtained is very encouraging and reveals the points to be improved at the level of our learning approach.

\section{INDEX}

Mots-clés : travaux pratiques, classe inversée, enseignement supérieur, expérimentation 


\section{AUTEURS}

\section{FATIMA LAKRAM}

Laboratoire STIC, Département de physique, Faculté des sciences, Université Chouaib Doukkali, El Jadida

fatima.lakrami@gmail.com

\section{OUIDAD LABOUIDYA}

Laboratoire STIC, Département de physique, Faculté des sciences, Université Chouaib Doukkali, El Jadida,

labouidya.o@ucd.ac.ma

\section{NAJIB ELKAMOUN}

Laboratoire STIC, Département de physique, Faculté des sciences, Université Chouaib Doukkali, El Jadida

elkamoun@gmail.com 